\title{
D-dimer is useful in the diagnosis of cortical venous sinus thrombosis
}

U sha K. M isra, Jayantee Kalita, Vikas Bansal

Department of Neurology, Sanjay Gandbi PGIMS, Lucknow, India

Address for correspondence:

Dr. U. K. Misra,

Department of Neurology,

Sanjay Gandhi Post Graduate

Institute of Medical Sciences,

Raebareilly Road,

Lucknow - 226 014, India.

E-mail: ukmisra@ sgpgi.ac.in /

drukmisra@ rediffmail.com

DOI: $10.4103 / 0028-3886.48822$

\section{Abstract}

Background: Estimations of D - dimer correlate with deep vein thrombosis and pulmonary thromboembolism and may serve as a marker of cortical venous sinus thrombosis (CVST). Aim: To study the usefulness of D-dimer in the diagnosis of CVST. M aterials and Methods: A total of 26 patients with CVST were subjected to a detailed medical history and neurological evaluation. A cranial M RI was carried out on a 1.5T scanner using T1, $T 2$, and DWI sequences and two-dimensional time of flight M R venography. $D$-dimer was estimated using a rapid latex agglutination slide test using monoclonal antibodies. Results: The age of patients ranged between 16 and 70 years old (median 31 years old); 8 were males and were examined after a mean duration of 22 days of symptoms. Cortical venous thrombosis was attributed to puerperium in 4 patients, infection in 5 patients, and pregnancy, dehydration, insect bite, and migraine in 1 patient each. Superior sagittal sinus was involved in 15 patients, transverse sinus in 16 patients, straight sinus in 3 patients, sigmoid sinus in 7 patients, and cavernous and deep system in 1 patient each. A total of 12 patients had more than one sinus involvement. D-dimer was positive in 20 patients and correlated with the duration of symptoms but not with the extent of sinus thrombosis and the outcome. Conclusion: D-dimer is useful in patients suspected of CVST and patients with positive test results should be urgently sent for MR imaging.

Key words: C ortical venous sinus thrombosis, D-dimer, M RI - M R venography

\section{Introduction}

Cortical venous sinus thrombosis (CVST) is a rare cause of headache and stroke but has a wide clinical spectrum, which includes headache, seizures, focal neurological signs, and alteration in consciousness in isolation or various combinations..$^{[1]}$ The reported incidence of CVST in the West is 3 to 4/million; whereas, in the developing countries it is reported to be $4.5 / 10000$ obstetric admissions. ${ }^{[2,3]}$ The gold standard for the diagnosis of CVST is magnetic resonance (MR) venography. A computed tomography (CT) scan has limitations, as it is positive in only $70 \%$ of the cases. ${ }^{[4]}$ In the developing countries where a majority of the cases with CVST occur, neuroimaging is either not available or beyond the reach of most patients. In such a situation, a biological marker, which is easy to carry out and affordable, is needed. D-dimer is a marker of endogenous fibrinolysis and should therefore be detectable in patients with deep vein thrombosis. Plasma levels of D-dimer, which are fragments of cross-linked fibrin degraded by plasmin, have been shown to be sensitive for the diagnosis of deep vein thrombosis and pulmonary thromboembolism. ${ }^{[5,6]}$ In the first report of D-dimer in MR venography confirmed CVST, all 6 patients had elevated levels of D-dimer using a qualitative bedside test; ${ }^{[7]}$ but in another study on 5 patients with CVST, 2 patients had D-dimer $<500 \mu \mathrm{g} / \mathrm{L}$ suggesting a lack of its sensitivity ${ }^{[8]}$ A number of other studies, however, have confirmed the usefulness of D-dimer in venous sinus thrombosis. ${ }^{[9,10]}$ Most of these studies are based on selected patient populations including patients with headache. ${ }^{[10,11]}$ We have evaluated consecutive patients with CVST with diverse clinical manifestations and highlight the importance of D-dimer in the diagnosis.

\section{Materials and $M$ ethods}

Consecutive patients diagnosed with CVST were 
asked to provide their medical history noting previous thromboembolic events, family history, and history of oral contraceptive or other hormonal preparations. The consciousness was assessed by Glasgow coma scale (GCS) score and cognitive function by Mini Mental state examination (MMSE). The presence of cranial nerve palsy and papilledema were noted. Muscle power was assessed on a 0-V MRC (Medical research Council) scale. Muscle tone, tendon reflex, joint position, pinprick, and cortical sensations were noted. The patients with CVST were categorized into those with headache, focal weakness, seizures, and altered sensorium. The severity of stroke was graded as per the Canadian Neurological Score (CNS) scale. ${ }^{[12]}$ A magnetic resonance imaging (MRI) was carried out on a 1.5 Tesla machine (Signa GE Medical system, Wisconsin, U.S.A.). Fast spin Echo T2, $\mathrm{T} 1$, and DW imaging were obtained in coronal and axial planes. The location, extent, and nature of abnormality were noted. The MR venography 2D-TOF/SPGR angiographic technique was used usually in two planes i.e., coronal and axial with an inferior saturation band to eliminate signals from arterial structures. Sections with a thickness of $1.5 \mathrm{~mm}$ were acquired in a coronal plane using the following parameters: 24/4.9(TR/TE), 50 degree flip angle, $210 \times 165 \mathrm{~mm}$ field of view, $256 \times 128$ matrix and 1 NEX (number of excitation). All MR venographic source images thus obtained were post-processed using the maximum (pixel) intensity projection (MIP) method, generating 12 MIP projection at 15 degree increments. We systematically reviewed the resulting venograms in each case for the presence of sinuses. Transverse sinus has a frequent normal variation, hence transverse sinus thrombosis was diagnosed only when T1 hyper intense signal on the MRI corresponding to a blood clot was obtained or there was evidence of collaterals. D-dimer level was done using the Latex Agglutination Slide Test (D-Di Kit-Diagnostica Stago, France) as per the manufacturer's recommendations. A total of $1.8 \mathrm{ml}$ of venous blood was collected in a vial containing $0.2 \mathrm{ml}$ of $3.8 \%$ solution of trisodium citrate. The sample was gently mixed and centrifuged at $3000 \mathrm{rpm}$ for 20 minutes and supernatant plasma was removed. After putting $20 \mathrm{ml}$ of plasma on the prescribed position on the test card, $20 \mathrm{ml}$ of a suspension of latex particles coated with anti D-dimer monoclonal antibody were mixed with it using a stirrer. The card was manually rotated for 3 minutes. Formation of visible clumps at the end of 3 minutes was taken as a positive result. ${ }^{[13]}$

\section{Results}

A total of 26 patients with CVST were recruited. Their median age was 31 years old (16-70 years old) and 8 patients were males. The precipitating events could be detected in half the patients, which included puerperium in 4 patients, pregnancy in 1 patient, infection in 5 patients, and dehydration, insect bite, and migraine in 1 patient each. Seventeen patients had altered sensorium and their GCS score ranged between 4 and 14 . Headache was present in all but 1 patient, vomiting was experienced in 22 patients, and focal motor deficit was present in 8 patients (hemiparesis in 7 and monoparesis in 1). Cranial nerve palsy was mainly in the form of $6^{\text {th }}$ cranial nerve palsy in 8 patients and total ophthalmoplegia in 1 patient who had cavernous sinus thrombosis. Seizures were present in 10 patients, of whom 3 had status epilepticus. MR venography revealed the involvement of superior sagittal sinus (SSS) in 15 patients, transverse sinus in 16 patients, straight sinus in 3 patients, sigmoid sinus in 7 patients, and cavernous sinus and deep venous system involvement in 1 patient each. Twelve patients had more than one cerebral venous sinus involvement and in 1 patient, all the superficial and deep sinuses were involved [Figure 1, Figure 2]. The duration of symptoms from the onset of symptoms ranged between 2 and 150 days (mean $=22$ days). At 3 months, 20 patients showed complete recovery, 4 patients showed a partial

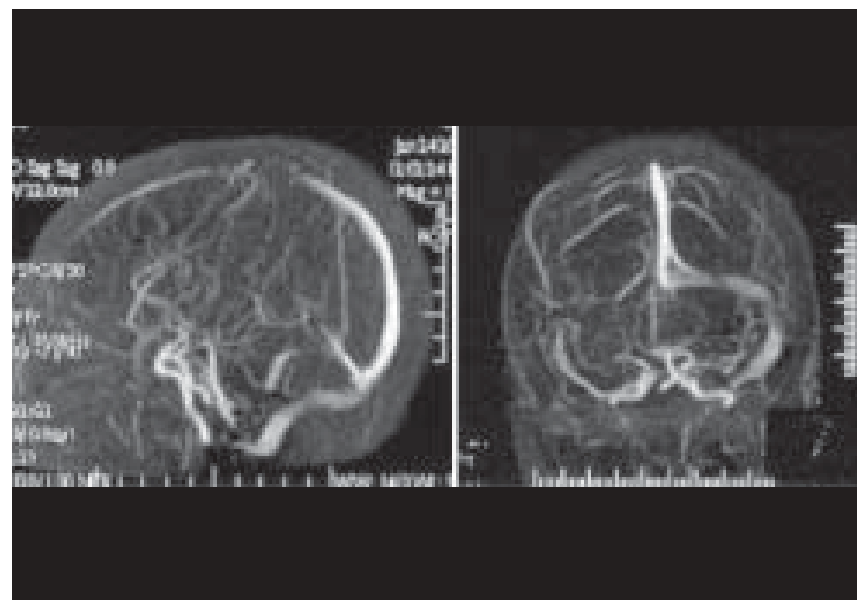

Figure 1: MR venography showing superior sagittal sinus and left transverse sinus thrombosis

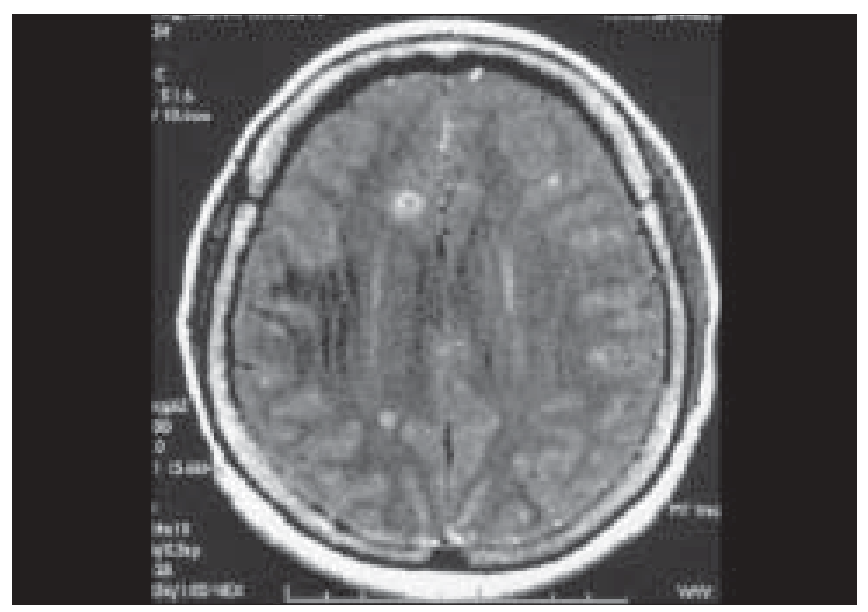

Figure 2: MRI of the same patient in T1 contrast axial section showing multiple ring-enhancing lesions with left parietal hypointensity 
recovery, and 2 patients showed poor recovery. The patients were treated with heparin subcutaneously for 10 days (conventional heparin was given to 6 patients, low molecular weight heparin was given to 20 patients) followed by oral anticoagulates and INR (international normalized ratio) was maintained between 2 and 2.5. Symptomatic therapies such as antiedema drugs (mannitol or carbonic enhydrase inhibitor) were given to 13 patients and anticonvulsants were prescribed to those with seizures. D-dimer was done within 24 hours of hospital admission. D-dimer was positive in 20 out of 26 patients. The sensitivity and specificity of D-dimer in the diagnosis of CVST was $77 \%$ each. The D-dimer was false negative in $23 \%$ of the patients.

D-dimer positivity did not correlate with the extent of venous sinus thrombosis $(P=0.93)$ and with 3 month outcome as assessed by the Barthel index score $(P=0.38)$ but inversely correlated with duration of illness $(P=0.01)$ [Figure 3]. The patients with positive D-dimer results had a shorter duration of illness (mean $=12$ days) compared with those with negative D-dimer results (mean $=76$ days). The patients' details are summarized in Table 1.

\section{Discussion}

Our study reveals that D-dimer results were positive in 20 out of 26 patients with CVST. These results are in agreement with the earlier report in which all 6 patients with CVST had positive D-dimer. ${ }^{[7]}$ All patient with CVST below 2 weeks had elevated D-dimer ${ }^{[9]}$ and Lalivi, et al. reported elevated D-dimer in 10 out of 12 CVST patients. ${ }^{[10]}$ Negative D-dimer in CVST patients could be attributed to a decrease of $\mathrm{D}$-dimer after a time period. In our patients, the duration of illness of D-dimer negative was much longer than the positive group (mean $=76 \mathrm{vs}$ 12 days). $D$-dimer levels tend to decrease progressively during the first week after a thromboembolic event and normalized within 3 months after deep vein thrombosis. ${ }^{[14]}$ In a study on 8 patients with CVST

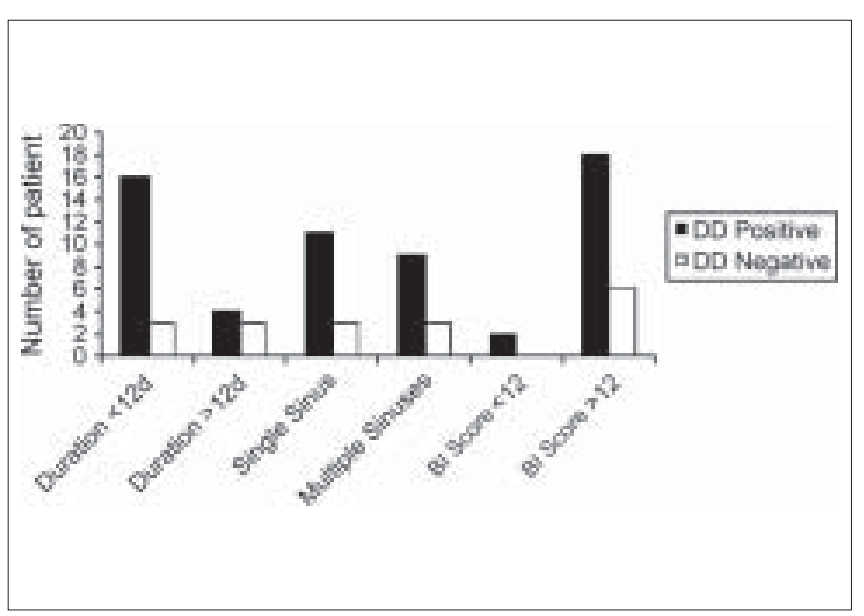

Figure 3: The relationship of clinical and radiological findings with D-dimer (DD) in patients with CVST. BI=Barthel index

\begin{tabular}{|c|c|c|c|c|c|c|}
\hline Age/Gender & Precipitating cause & Clinical syndrome & Sinuses involved & D-dimer & Day of investigation & $\begin{array}{c}\mathrm{BI} \\
\text { Score } \\
\end{array}$ \\
\hline 65/F & - & Altered sensorium & SSS & + & 15 & 20 \\
\hline $50 / \mathrm{F}$ & Infection & Altered sensorium & SSS+Lat S & + & 19 & 4 \\
\hline 16/F & - & Altered sensorium & SSS & - & 20 & 20 \\
\hline $26 / F$ & Puerperium & Seizure & SSS & + & 8 & 20 \\
\hline $23 / \mathrm{F}$ & Puerperium & Stroke & SSS & + & 4 & 18 \\
\hline $25 / F$ & Puerperium & Altered sensorium & Lat S & + & 45 & 20 \\
\hline 60/F & - & Stroke & Lat S & + & 10 & 6 \\
\hline $35 / \mathrm{F}$ & Pregnancy & Stroke & SSS+Lat S+St & + & 5 & 20 \\
\hline 70/F & Infection & Altered sensorium & Lat S+Sig & + & 25 & 0 \\
\hline $22 / F$ & - & Altered sensorium & Lat S+St & + & 4 & 18 \\
\hline $32 / F$ & - & Altered sensorium & All & - & 30 & 20 \\
\hline $45 / F$ & Infection & Altered sensorium & Lat S+Sig & + & 28 & 20 \\
\hline $28 / F$ & - & Headache & SSS & - & 150 & 20 \\
\hline $42 / F$ & Dehydration & Stroke & SSS+Sig & + & 4 & 20 \\
\hline $45 / F$ & - & Encephalopathy & 「 & + & 5 & 20 \\
\hline 23/M & - & Seizure & SSS+Lat S & + & 8 & 20 \\
\hline 40/M & Infection & Encephalopathy & Lat S+Sig & + & 15 & 20 \\
\hline 16/M & - & Headache & SSS & + & 6 & 20 \\
\hline 65/F & - & Stroke & SSS+Lat S & - & 6 & 18 \\
\hline $25 / \mathrm{M}$ & - & Stroke +seizure & SSS+Lat S & + & 7 & 20 \\
\hline $23 / F$ & Puerperium & Stroke +seizure & Lat S & + & 38 & 20 \\
\hline 39/F & - & Headache & Lat S & + & 17 & 20 \\
\hline 16/M & Infection & Stroke +seizure & All superficial & - & 21 & 20 \\
\hline 44/M & Migraine & Headache +seizure & Lat S & + & 3 & 20 \\
\hline 30/M & Insect bite & Painful ophthalmoplegia & Cavernous & + & 3 & 12 \\
\hline 22/M & - & Headache +stroke & SSS & - & 18 & 20 \\
\hline
\end{tabular}

SSS = superior sagittal sinus; Lat $\mathrm{S}=$ lateral sinus; $\mathrm{St}$ = straight sinus; $\mathrm{Sig}=$ sigmoid sinus; $\mathrm{Bl}=\mathrm{Barthel}$ index 
with a duration ranging from 1 to 4 months, 6 patients had a D-dimer level in the reference range suggesting a possible correlation between duration of disease and $\mathrm{D}$-dimer levels. ${ }^{[15]} \mathrm{D}$-dimer is a marker of endogenous fibrinolysis, therefore, it was expected to correlate with the extent of venous thrombosis. However, in our study, there was no relation between the D-dimer level and the extent of CVST. Of the 6 patients with absent D-dimer, 2 had thrombosis of all the cerebral venous sinuses. A lack of relationship between D-dimer level and the extent of venous thrombosis was also reported by others. ${ }^{[10]}$ The magnitude of thrombosis expressed as the number of sinuses and veins involved tended to be more imperfect in patients with neurological deficit in comparison with the patients presenting with headache only. Moreover, the patients with a deficit were prone to a higher D-dimer level; however, the difference was not statistically significant. ${ }^{[10]}$ We could not correlate D-dimer level with extent of thrombosis because we carried out a qualitative test for D-dimer.

The diversity in the types of tests available for D-dimer test raises the question of choosing the best test. The first report of D-dimer in CVST was based on qualitative bedside test. ${ }^{[7]}$ We have estimated D-dimer using the rapid latex slide agglutination method. The high efficacy of this method was reported in a comparative study. ${ }^{[16]}$ The sensitivity of the D-dimer test ranged between $81 \%$ and $95 \%{ }^{[17,18]}$ and specificity ranged between $22 \%$ and $61 \%{ }^{[19,20]}$ in patients with deep vein thrombosis using the latex slide agglutination method using the Diagostica Stago kit. There are various assays available but the one that we have used is reliable and cost effective. Quantitative assays of D-dimer estimation have become available recently but we could not use them for lack of availability in our setup.$^{[10,11]}$ More elaborate methods with quantification are available. The tests have a comparable sensitivity and specificity. ${ }^{[21]}$ In a study conducted by Cucchiara, et al., a D-dimer level $<500 \mathrm{ng} \mathrm{mL}^{-1}$ using the VIDAS assay or $<0.5 \lg \mathrm{FEU} \mathrm{mL}^{-1}$ using the MDA assay reliably ruled out the presence of CVST. All 9 confirmed patients with CVST had D-dimer above these levels. ${ }^{[22]}$

D-dimer tests can have false positivity. Although we have not carried out D-dimer testing in normal controls, elevated levels have been reported in inflammatory and infectious disorders. ${ }^{[10]} \mathrm{A}$ total of 4 out of 42 patients in the control group had elevated levels of D-dimer, which were attributed to pregnancy, rheumatoid arthritis, and meningitis. ${ }^{[23-25]}$ In a study conducted by Kosinski, et al., 34 out of 35 patients with CVST had high D-dimer levels giving a negative predictive value of 99.6\%, specificity of $91.2 \%$, and a positive predictive value of $55.7 \% \cdot{ }^{[11]}$ With the current information, clinically suspected patients with CVST whose D-dimer test is positive should undergo urgent MR venography for confirmation. However, more experience is needed before formulating a recommendation for practice.

\section{References}

1. Cakmak S, Derex L, Berruyer M, Nighoghossian N, Philippeau F, Adeleine $\mathrm{P}$, et al. Cerebral venous thrombosis Clinical outcome and systematic screening of prothrombotic factors. Neurology 2003;60:1175-8.

2. Stam J. Thrombosis of the cerebral veins and sinuses. N Engl J Med 2005;352:1791-8.

3. Bansal BC, Gupta RR, Prakash C. Stroke during pregnancy and puerperium in young females below the age of 40 years as a result of cerebral venous/venous sinus thrombosis. Jpn Heart J 1980;21:171-83.

4. Bousser MG. Cerebral venous thrombosis: Diagnosis and management. J Neurol 2000;247:252-8.

5. Bounameaux H, de Moerloose P, Perrier A, Reber G. Plasma measurement of D-dimer as diagnostic aid in suspected venous thromboembolism: An overview. Thromb Haemost 1994;71:1-6.

6. Brill-Edwards P, Lee A. D-dimer testing in the diagnosis of acute venous thromboembolism. Thromb Haemost 1999;82:688-94.

7. Wildberger JE, Mull M, Kilbinger M, Schon S, Vorwerk D. Cerebral sinus thrombosis: Rapid test diagnosis by demonstration of increased plasma D-dimer levels (simpliRED). Rofo Fortschr Geb Rontgenstr Neuen Bildgeb Verfahr 1997;167:527-9.

8. Talbot K, Wright M, Keeling D. Normal D-dimer levels do not exclude the diagnosis of cerebral venous sinus thrombosis. J Neurol 2002;249:1603-4.

9. Tardy B, Tardy-Poncet B, Viallon A, Piot M, Garnier P, Mohamedi R, et al. D-dimer levels in patients with suspected acute cerebral venous thrombosis. Am J Med 2002;113:238-41.

10. Lalive PH, de Moerloose P, Lovblad K, Sarasin FP, Mermillod B, Sztajzel R. Is measurement of D-dimer useful in the diagnosis of cerebral venous thrombosis? Neurology 2003;61:1057-60.

11. Kosinski CM, Mull M, Schwarz M, Koch B, Biniek R, Schläfer J, et al. Do normal D-dimer levels reliably exclude cerebral sinus thrombosis? Stroke 2004;35:2820-5.

12. Cote R, Hachinski VC, Shurrell BL, Norris BW, Wolfson C. The Canadian Neurological Score: A preliminary study. Stroke 1986;17:731-7.

13. Gupta D, Gupta S, Balamugesh T, Aggarwal AN, Das R. Circulating D-dimers as a marker of disease activity in pulmonary sarcoidosis. Indian J Chest Dis Allied Sci 2005;47:175-9.

14. Sie P, Cadroy Y, Elias A, Boccalon H, Boneu B. D-dimer levels in patients with long term antecedents of deep venous thrombosis. Thromb Haemost 1994;72:161-2.

15. Quattrone A, Gambardella A, Carbone AM, Oliveri RL, Lavano A, De Marco EV, et al. A hypofibrinolytic state in overweight patients with cerebral venous thrombosis and isolated intracranial hypertension. J Neurol 1999;246:1086-9.

16. Gupta PK, Gupta M, Chatterjee T, Saxena R. Comparative evaluation of whole blood D-dimer test to plasma D-dimer test for diagnosis of disseminated intravascular coagulation. Indian J Exp Biol 2005;43:382-4.

17. Wijns W, Daoud N, Droeshout I, Pradier O, Wautrecht JC, Golzarian J, et al. Evaluation of two D-dimer assays in the diagnosis of venous thromboembolism. Acta Clin Belg 1998;53:270-4.

18. Legnani C, Pancani C, Palareti G, Guazzaloca G, Fortunato G, Grauso F, et al. Comparison of new rapid methods for D-dimer measurement to exclude deep vein thrombosis in symptomatic outpatients. Blood Coagul Fibrinolysis 1997;8:296-302.

19. Elias A, Aptel I, Huc B, Chale JJ, Nguyen F, Cambus JP, et al. D-Dimer test and diagnosis of deep vein thrombosis: A comparative study of 7 assays. Thromb Haemost 1996;76:518-22.

20. Funfsinn N, Caliezi C, Biasiutti FD, Korte W, Z'Brun A, Baumgartner I, et al. Rapid D-dimer testing and pre-test clinical probability in the exclusion of deep venous thrombosis in symptomatic outpatients. Blood Coagul Fibrinolysis 2001;12:165-701.

21. Janssen MC, Heebels AE, de Metz M, Verbruggen H, Wollersheim H, Janssen S, et al. Reliability of five rapid D-dimer assays compared to 
ELISA in the exclusion of deep venous thrombosis. Thromb Haemost 1997;77:262-6.

22. Cucchiara B, Messe S, Taylor R, Clarke J, Pollak E. Utility of D-dimer in the diagnosis of cerebral venous sinus thrombosis. J Thromb Haemost 2005;3:387-9.

23. Chabloz P, Reber G, Boehlen F, Hohlfeld P, de Moerloose. TAFI antigen and D- dimer levels during normal pregnancy. Br J Haematol 2001;115:150-2.

24. Beckham JC, Caldwell DS, Peterson BL, Pippen AM, Currie MS, Keefe FJ, et al. Disease severity in rheumatoid arthritis: Relationships of plasma tumor necrosis factor alpha, soluble interleukin 2- receptor; soluble CD4/CD8 ratio, neopterin and fibrin D-dimer to traditional severity and functional measures. J Clin Immunol 1992;12:353-61.

25. Ben RJ, Feng NH, Ku CS. Meningoencephalitis, myocarditis and disseminated intravascular coagulation in a patient with scrub typhus. J Microbiol Immunol Infect 1999;32:57-62

Accepted on 24-02-2009

Source of Support: Nil, Conflict of Interest: None declared.

\section{Author Help: Reference checking facility}

The manuscript system (www.journalonweb.com) allows the authors to check and verify the accuracy and style of references. The tool checks the references with PubM ed as per a predefined style. Authors are encouraged to use this facility, before submitting articles to the journal.

- $\quad$ The style as w ell as bibliographic elements should be $100 \%$ accurate, to help get the references verified from the system. Even a single spelling error or addition of issue number/month of publication will lead to an error when verifying the reference.

- Example of a correct style

Sheahan P, O'leary G, Lee G, Fitzgibbon J. Cystic cervical metastases: Incidence and diagnosis using fine needle aspiration biopsy. Otolaryngol Head Neck Surg 2002;127:294-8.

- Only the references from journals indexed in PubM ed will be checked.

- $\quad$ Enter each reference in new line, without a serial number.

- $\quad$ Add up to a maximum of 15 references at a time.

- If the reference is correct for its bibliographic elements and punctuations, it will be shown as CORRECT and a link to the correct article in PubMed will be given.

- If any of the bibliographic elements are missing, incorrect or extra (such as issue number), it will be shown as INCORRECT and link to possible articles in PubM ed will be given. 